\title{
AUTOREGRESSIVE MODELING OF GOLF VELOCITY TIME SERIES
}

\author{
R. ULRICH, F. VARADI, L. BERTELLO \\ University of California, Los Angeles
}

AND THE GOLF TEAM

The frequencies of solar p-modes are usually computed by fitting Lorentzian profiles to the observed signals' Fourier spectra. We offer a new technique to estimate mode parameters: direct modeling by autoregressive stochastic processes, in conjunction with Singular Spectrum Analysis.

A second order autoregressive (AR) stochastic process regresses the signal $y_{i}$ on its previous values and it is defined by the second order difference equation $y_{i}=a_{1} y_{i-1}+a_{2} y_{i-2}+w_{i}$, where $w_{i}$ is excitation, assumed to be white noise. This is a forced and damped oscillator in discrete time whose frequency and damping factor are related to the model coefficients $a_{1}$ and $a_{2}$ by the characteristic equation. It is also a filter with input $w_{i}$ and output $y_{i}$ and its spectral response has Lorentzian profile. These and the autoregressive-moving average (ARMA) models are the mainstay of digital signal processing and spectral analysis (Proakis et al., 1992). When additive noise contaminates the signal, the correct model would be ARMA, but it is difficult to fit.

We apply Singular Spectrum Analysis (SSA, Vautard et al., 1992) to extract the AR part of the signal. In SSA, one diagonalizes the autocovariance matrix (considered up to a certain lag, the window length) to obtain its eigenvalues (the singular spectrum) and eigenvectors. The latter are used as filters to isolate various components of the signal. The singular spectrum usually exhibits a noise floor, and the components above it tend to be associated with modes. We use these filtered signals above the noise to fit AR models.

We apply the technique to 370 days of the velocity time series provided by the GOLF experiment (Gabriel et al., 1997). Since SSA cannot deal with thousands of modes, we extract and resample the signal in frequency bands which contain particular modes, yielding a few hundred points in the time domain. Three different widths for these bands and for each band a different SSA window length are used. AR models of orders corresponding to the expected rotational splitting are fitted using both Burg's method and the least-squares modified Yule-Walker equations. 
Singular Spectra of the $I=2, n=13$ mode

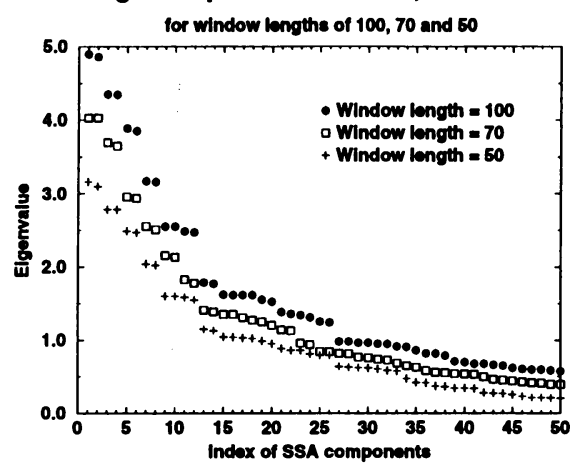

Rotational splitting of the $l=2, n=13$ mode

meane and ranges of 24 AR eetimates for each index

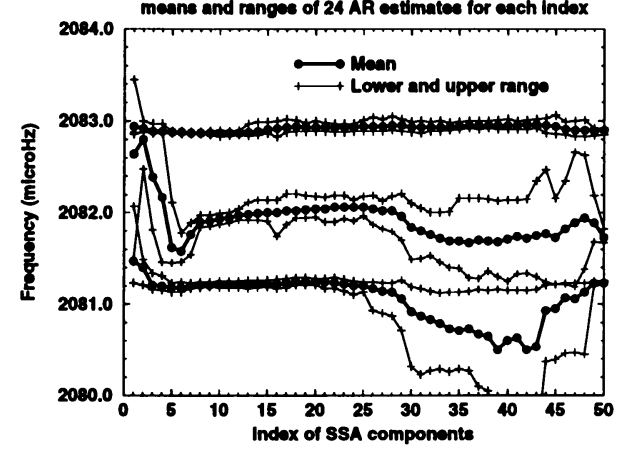

Figure 1. Singular Spectra and AR frequency estimates for the $l=2, n=13$ p-mode.

The left panel of Fig. 1 shows the singular spectra for a mode. The first 15 or so SSA components appear to be significant and the rest form the noise floor. We reconstructed the signal from the first component up to the 50th and fitted AR models for each reconstruction. The right panel shows the means and ranges of the estimated frequencies. They are fairly stable around the index which marks the beginning of the noise floor - between 10 and 20 . We obtained estimates of both frequency and rotational splitting for several $l=1$ and $l=2$ modes and for each mode we averaged the stable cases near the beginning of their respective noise floors. These results are in good agreement with maximum likelihood estimates (MLE) of Lorentzian fits (see Table 1).

\begin{tabular}{llllll}
\hline & \multicolumn{2}{c}{ MLE+Lorentzian } & \multicolumn{2}{c}{ AutoRegressive } \\
$l$ & $n$ & Frequency $(\mu \mathrm{Hz})$ & Splitting $(\mathrm{nHz})$ & Frequency $(\mu \mathrm{Hz})$ & Splitting $(\mathrm{nHz})$ \\
\hline 1 & 10 & $1612.75 \pm 0.03$ & $425 \pm 33$ & $1612.71 \pm 0.05$ & $400 \pm 50$ \\
1 & 11 & $1749.31 \pm 0.03$ & $412 \pm 27$ & $1749.31 \pm 0.05$ & $410 \pm 50$ \\
1 & 12 & $1885.11 \pm 0.03$ & $405 \pm 27$ & $1885.12 \pm 0.03$ & $425 \pm 30$ \\
1 & 13 & $2020.82 \pm 0.04$ & $467 \pm 32$ & $2020.85 \pm 0.04$ & $485 \pm 40$ \\
1 & 14 & $2156.76 \pm 0.06$ & $415 \pm 43$ & $2156.76 \pm 0.07$ & $445 \pm 70$ \\
1 & 15 & $2292.04 \pm 0.07$ & $424 \pm 60$ & $2292.02 \pm 0.09$ & $400 \pm 90$ \\
2 & 12 & $1945.79 \pm 0.03$ & $370 \pm 17$ & $1945.81 \pm 0.07$ & $395 \pm 70$ \\
2 & 13 & $2082.06 \pm 0.05$ & $391 \pm 26$ & $2082.08 \pm 0.09$ & $425 \pm 90$ \\
\hline
\end{tabular}

TABLE 1. Mode parameters.

\section{References}

Gabriel, A. H. et al.: 1997, 'Performance and early results from the GOLF instrument flown on the SOHO mission', Solar Physics, in press

Proakis, J. G., Rader, C. M. Ling, F., and Nikias, C. L.: 1992, Advanced Digital Signal Processing, Macmillan Publ. Co.

Vautard, R., Yiou, P. and Ghil, M.: 1992, 'Singular-spectrum analysis: A toolkit for short, noisy chaotic signals,' Physica $D$ 58, 95-126 